\title{
Observation of symmetric patterns in the flux distribution of thin $\mathrm{YBa}_{2} \mathrm{Cu}_{3} \mathrm{O}_{7-x}$ films
}

\author{
P. Brüll, D. Kirchgässner and P. Leiderer \\ Universität Konstanz, D-7750 Konstanz, Germany
}

Reccived 22 August 1991

Revised manuscript received 13 September 1991

\begin{abstract}
We have investigated the magnetic field distribution in epitaxial $\mathrm{YBa}_{2} \mathrm{Cu}_{3} \mathrm{O}_{7} x$ films on square $\mathrm{SrTiO}_{3}$ and $\mathrm{MgO}$ substrates. In fields between $20 \mathrm{mT}$ and IT a magnetooptic method was applied.complemented by a scanning micro Hall probe for the low field range. The flux is observed to penetrate in a rather regular way, displaying the fourfold symmetry of our samples. The penetration starts preferentially from the sampie edges. A qualitative description of this penetration process is suggested, which relies on the curvature of the shielding current paths.
\end{abstract}

\section{Introduction}

Among the potential applications of high-Te superconductors thin epitaxial films of these materials will certainly play a prominent role. For this purpose it is essential to have methods available which allow one to check the sample quality on a large scale. simultaneously being fast and contactless. Here we report on measurements with a magneto-optical technique which fulfills these requirements.

We have used this method to study the penetration of magnetic flux and the resulting distribution in square $\mathrm{YBa}_{2} \mathrm{Cu}_{3} \mathrm{O}_{7-x}$ films in fields up to $1 \mathrm{~T}$. The measurements show that - in contrast to what one might have expected - the flux starts to penetrate not at the sample corners, but rather in the middle of the edges of the square. Recently there have been several reports about similar investigations [1, 2], although these studies were mainly performed on a smaller spatial scale, ranging from some $10 \mu \mathrm{m}$ up to $1-2 \mathrm{~mm}$. Our experiments covered the whole sample area of $10 \times 10 \mathrm{~mm}^{2}$. They demonstrate that for a homogeneous film one obtains well defined symmetric flux patterns, which reflect the fourfold symmetry of our samples. Imperfections like scratches or inhomogeneities of the films are readily visible. In the low field range $\left(\simeq 10^{-2} \mathrm{~T}\right)$ the magnetooptical experiments were complemented by measurements with a scan- ning micro Hall probe, which confirmed these results.

\section{Experiment}

The epitaxial $\mathrm{YBa}_{2} \mathrm{Cu}_{3} \mathrm{O}_{7-x}$ films investigated here were laser ablated onto $10 \times 10 \mathrm{~mm}^{2}(001) \mathrm{SrTiO}_{3}$ substrates [3] or evaporated on (100) $\mathrm{MgO}$. The films on $\mathrm{SrTiO}_{3}$ had a thickness of about $300 \mathrm{~nm}$, those on $\mathrm{MgO} 100 \mathrm{~nm}$ and $150 \mathrm{~nm}$, respectively. The critical current density of all films was about $10^{6} \mathrm{~A} /$ $\mathrm{cm}^{2}$ at $77 \mathrm{~K}$.

The measurements were performed in an optical toploading cryostat down to temperaturcs of $2 \mathrm{~K}$ Images of the flux distribution were obtained by mcans of the Faraday rotation of a thin EuS film $[4,5]$. In contrast to the experiment of ForkJ et al. [2] the EuS (without addition of EuF) was not evaporated directly onto the sample, but instead onto a glass substrate, and was subsequently covered with an aluminum film in order to increase the reflectance for the light to be analyzed [6]. This leaves the surface of the superconductor in its original state, an important requirement for future characterization purposes.

Since the magnetooptic experiments were carried out with a HeNe laser, the thickness of the EuS film was optimized with respect to this light source by a 
method first introduced by Kirchner [4]. Very good contrast is obtained if the thickness of the EuS film is $\frac{5}{4} \lambda$, where $\lambda$ is the wavelength of a HeNe laser in the EuS film.

The glass with the sandwich of EuS and aluminum was directly placed on top of the $\mathrm{YBa}_{2} \mathrm{Cu}_{3} \mathrm{O}_{7-x}$ film. In all experiments the superconducting sample and the glass with the EuS film were immersed in superfluid helium. The EuS film was magnetooptically active up to a temperature of $T \simeq 12 \mathrm{~K}$, somewhat lower than the ferromagnetic Curie temperature of $16.9 \mathrm{~K}$ reported for this material [4]. This may be due to an excess of Eu, as measured by EDS (energy dispersive $X$-ray spectrometry), and also to impurity atoms. To check the possible existence of ferromagnetic domains $[7,8]$ we performed a magnetooptic experiment, as described above, without a superconductor in external fields up to $1 \mathrm{~T}$. No structures on a submillimeter range, indicating the existence of a domain pattern in the EuS film, were observed both with the external field applied as well as after reducing it to zero. Therefore we exclude the influence of ferromagnetic domains on the observed magnetic field distributions in the superconductor, at least in the range of our resolution.

The sensitivity of the magnetooptic set-up used here was limited to magnetic fields $\geq 20 \mathrm{mT}$. Therefore we investigated the low field range below $20 \mathrm{mT}$ in a complementary experiment. A scanning micro Hall probe at a spatial resolution of about $0.04 \mathrm{~mm}^{2}$ allowed one to obtain field distributions across the whole sample surface by performing $x y$-scans. Although much coarser than the magnetooptic technique, this method provided a useful extension and verification of the optical results.

\section{Results}

Figure $1(a-c)$ shows a set of pictures taken during the increase of the external field up to $0.25 \mathrm{~T}$ after zero field cooling (ZFC). The magnetic field was applied in a direction perpendicular to the sample surface. Bright areas in these pictures correspond to regions of high magnetic field. The spatial resolution in the present experiment was $50 \mu \mathrm{m}$, a value which apparently can be further improved by proper imaging optics.
In fig. 1 (a) the flux expulsion leads to an increase in the brightness of the sample edges. It is important to note that the magnetic field is maximal around the centers of the edges rather than in the corners. On a rough scale, the flux penetrates the film in a uniform way from all four sides simultaneously establishing overall concave flux fronts which display structures in the submillimeter range. The concave flux penetration from the edges of the sample yields a well-shielded region along the diagonals, giving rise to a roughly cross-like pattern of the same fourfold symmetry as the sample (fig. 1(b)). The width of this pattern shrinks as the field is increased, until at $0.25 \mathrm{~T}$ it is only a few tenths of a $\mathrm{mm}$ wide (fig. 1 (c)). At higher fields shielding in this region gradually vanishes, until above $0.5 \mathrm{~T}$ we could not detect any influence of the superconductor in our pictures. These fields also lead to a saturation in the remanent flux distribution, which remains unchanged even after applying fields as high as $1 \mathrm{~T}$.

The remanent flux distribution after applying 0.25 $T$ is shown in fig. 1 (d). The largest amount of trapped flux is observed around the region which formerly displayed the best shielding.

To make sure that all the features described above correspond to the superconducting sample (and not to the EuS film) we have performed a second run with the sample being rotated by an angle of $90^{\circ}$. The results were identical to the previous ones, but the images were rotated in the expected manner.

In fig. 2 we show for comparison results for a second sample which appears to be less homogeneous. Figure $2(a-c)$ again presents the increase of the field from zero to $0.2 \mathrm{~T}$ after $\mathrm{ZFC}$. As before the flux fronts are found to penetrate the sample from the edges, and the best shielded regions are again roughly along the diagonals of the sample. Apparently the flux penetrates preferentially from the right side, and has a more inhomogeneous distribution compared to sample 1. The inhomogeneities lead to a distortion of the shielded cross-shaped structure. The remanent flux distribution after reducing the field to zero is shown in fig. 2(d). Again the largest amount of flux is trapped around the regions which were formerly the best shielded ones.

Results for the second sample in the low field region, obtained with the Hall probe, are presented in fig. 3. The sample was again cooled in zero field, and 

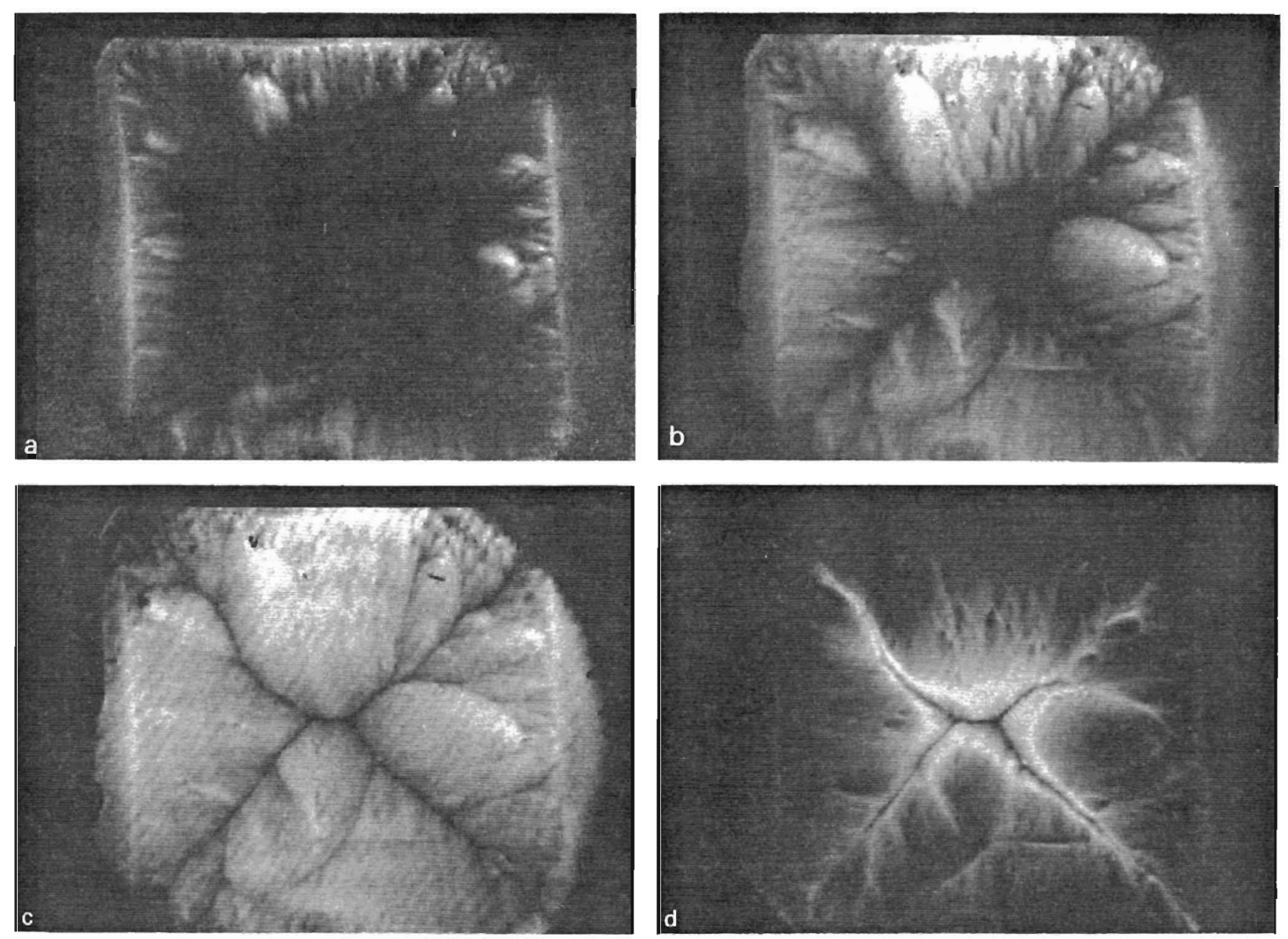

Fig. 1. Flux distribution in an epitaxial $300 \mathrm{~nm}$ thick $\mathrm{YBa}_{2} \mathrm{Cu}_{3} \mathrm{O}_{7-x}$ film on a $10 \times 10 \mathrm{~mm}^{2}(001) \mathrm{SrTiO}_{3}$ substrate (sample I). The sample was cooled in zero field and the pictures were taken during increase of the field up $10 B=0.25 \mathrm{~T}$ (which lasted 2 min). The light intensity distribution corresponds to the magnetic field distribution. The contour of the square film is outlined by the expelled flux. best visible in fig. 1 (a). The applied field was (a) $B=0.06 \mathrm{~T}$, (b) $0.12 \mathrm{~T}$, (c) $0.25 \mathrm{~T}$, (d) remanent magnetization. $B=0$.

then exposed to an external field of $1 \mathrm{mT}$ and $19 \mathrm{mT}$ respectively. The curves represent lines of constant field at a height of $0.3 \mathrm{~mm}$ above the sample surface, plotted at intervals of $0.1 \mathrm{mT}$ in fig. 3 (a) and $1 \mathrm{mT}$ in (b). The location of the sample is indicated by the square. It should be noted that the field values near the centers of the edges exceed the externally applied field by about 10\% whereas the field above the corners of the sample is nearly identical to the external field. The inhomogeneity of the sample pointed out already in fig. 2 is hardly discernible at $1 \mathrm{mT}$ but leads to quite a pronounced distortion in a field of $19 \mathrm{mT}$ at the right side of fig. $3(\mathrm{~b})$.

\section{Discussion}

The observed overall patterns are obviously a geometric effect, to be explained by the square symmetry of our samples. The pictures are reminescent of a magnetooptic experiment by Huebener et al. [6], where rectangular $\mathrm{Nb}$-foils and -films displayed similar structures. There the structure was attributed to domains with different direction of magnetization, comparable to ferromagnets.

For the structures observed here in $\mathrm{YBa}_{2} \mathrm{Cu}_{3} \mathrm{O}_{7-x}$ films this explanation can be ruled out: if there were domains the magnetization in neighboring areas would have to be of opposite polarity. This was not the case, as could be shown by determining the sign 

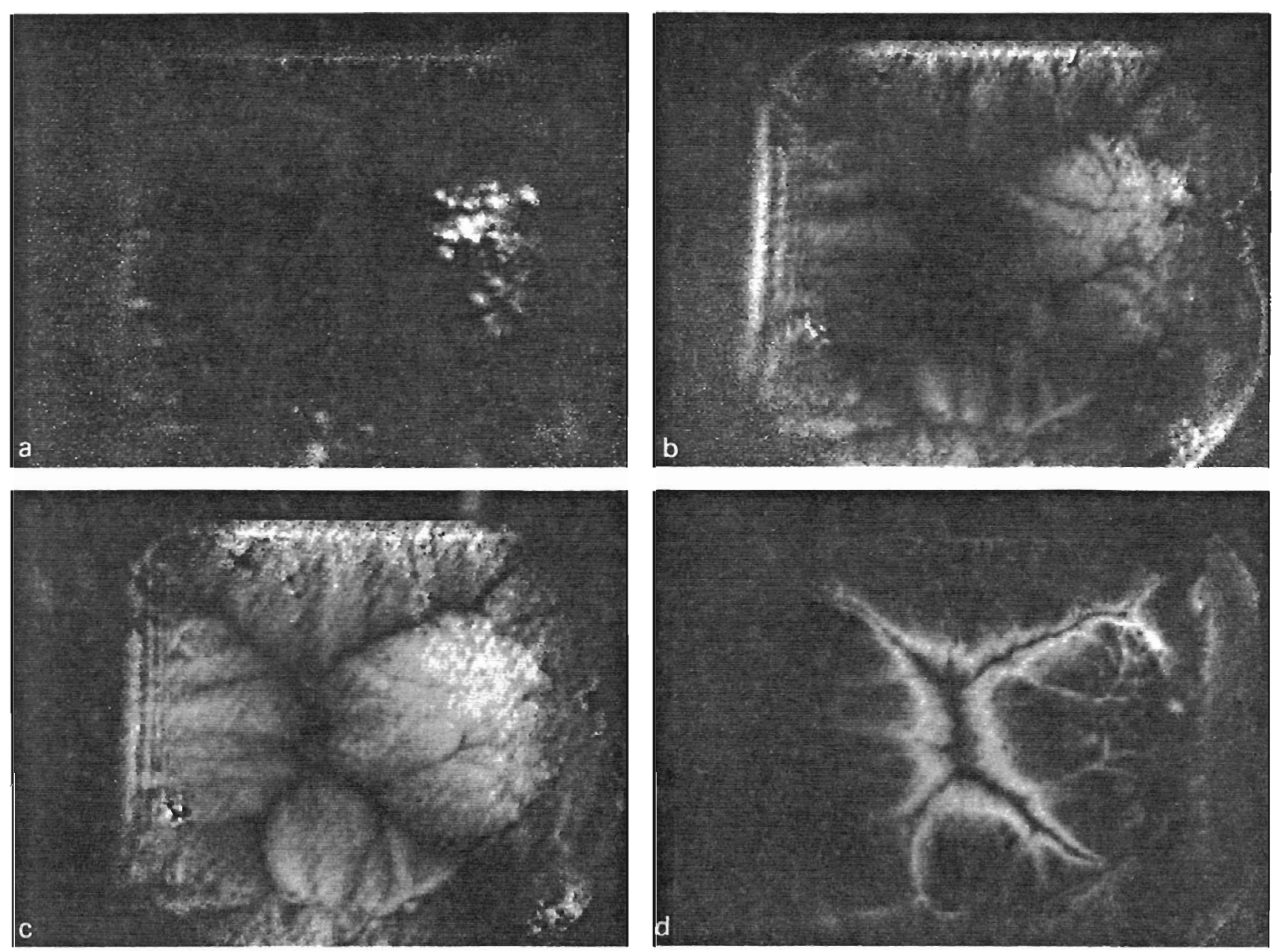

Fig. 2. Flux distribution in an epitaxial $300 \mathrm{~nm}$ thick $\mathrm{YBa}_{2} \mathrm{Cu}_{3} \mathrm{O}_{7-x}$ film on a $10 \times 10 \mathrm{~mm}^{2}$ (00I) $\mathrm{SrTiO}_{3}$ substrate (sample 2). The sample was cooled in zero field and the pictures were taken during increase of the field up to $B=0.2 \mathrm{~T}$. (a) $B=0.04 \mathrm{~T}$, (b) $0.12 \mathrm{~T}$, (c) $0.2 \Upsilon$. (d) remanent magnetization, $B=0$.

of the magnetization with magnetooptics (turning the analyzer). In addition the measurements with the Hall probe clearly revealed that the sign is the same in all four quadrants, both for shielding and for the remanent magnetization.

In our interpretation the local variations in the field distribution resulting from the flux expulsion are responsible for the occurrence of the cross-like structures. As was noted in the discussion of fig. 1 (a) and the $x y$-scan with the Hall probe (fig. 3), the field appears to be maximal around the centers of the sample edges. Therefore this is the location where the lower critical field is reached first, giving rise to the observed concave flux fronts penetrating the sample. Consequently the shielding current path is deformed as sketched schematically in fig. 4 , where in addition the field generated by the shielding currents is represented by small circles. The shielding efficiency of the currents is obviously dependent on the sign as well as on the magnitude of their curvature. As suggested by fig. 4 the shielding of the corners is better than in the middle of the edges. Moreover it is seen that on the outside of the current path, where the field of the shielding current adds to the external field, the largest enhancement is expected near the middle of the edges, as observed experimentally. The persistence of sharp structures along the sample diagonals, pronouncedly visible in figs. 1 (c) and $2(\mathrm{c})$, can in this picture be explained by the strong magnetic repulsion of two current paths with opposite direction. 

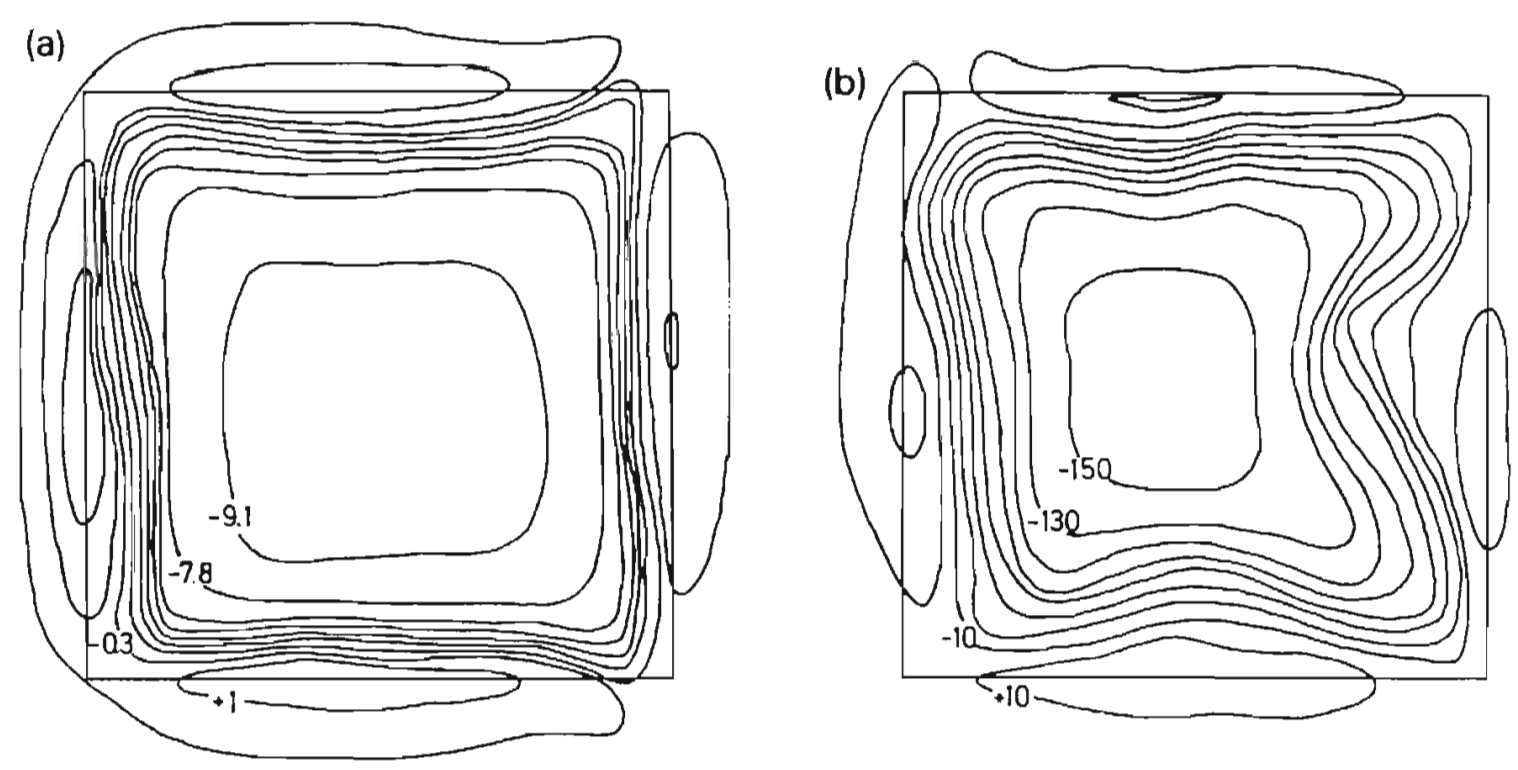

Fig. 3. Field distribution of sample 2 in the low field range obtained with a scanning micro Hall probe. The curves indicate lines of constant fietd at a height of $0.3 \mathrm{~mm}$ above the sample surface. (a) $B=1 \mathrm{mT}$. (b) $B=19 \mathrm{mT}$. The numbers refer to the difference between the local magnetic field and the externally applied field (in $\mathrm{mT}$ ).

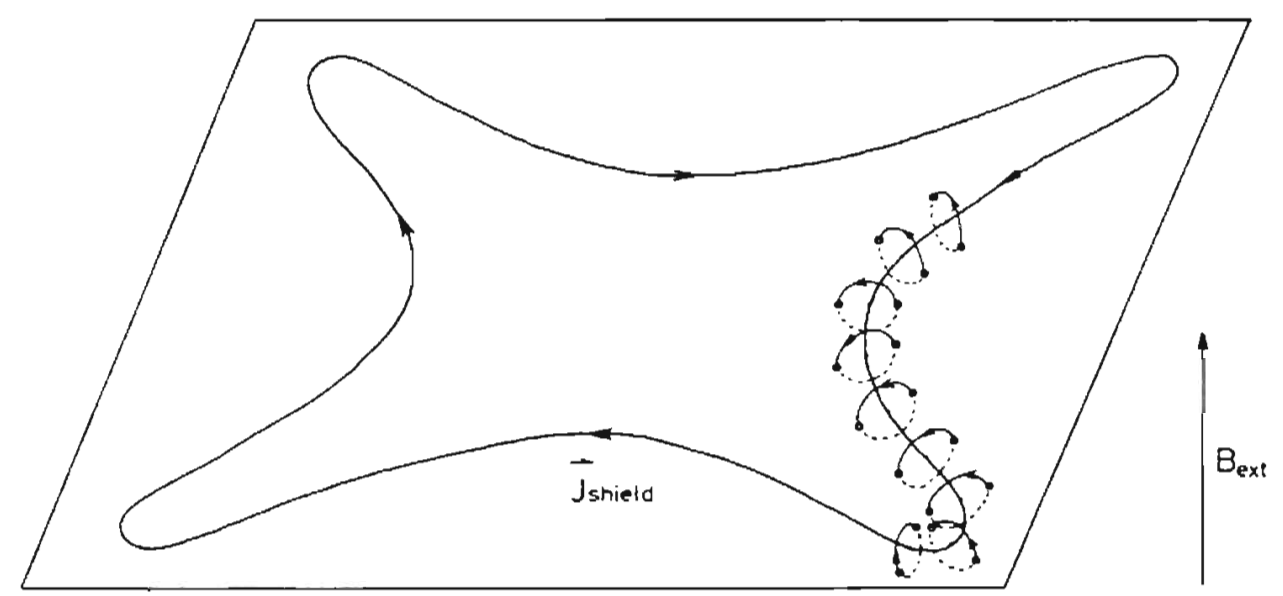

Fig. 4. Schematic sketch of the current path in an intermediate magnetic field where the flux has already started to penetrate the sample. The small circles represent the magnetic field that is generated by the current.

A more quantitative description taking into account the different components of rot $B=\mu_{0} j$, as was reported by Baczewski et al. [9], is in preparation.

Inhomogeneities in the sample give rise to a deviation of the shielding structure from a perfect cross. We have investigated several samples with a varying degree of homogeneity in the superconducting quality. The according degree of distortion of the cross was clearly related to the overall sample homoge- neity, not taking into account structures on a submillimeter scale. The appearance of the pattern does not depend on the substrate, nor on the preparation process, as can be seen in fig. 5 for a thermally evaporated film on $\mathrm{MgO}$. Altogether one can thus take the deviation of the magnetooptic pattern from the fourfold sample symmetry as a measure of the overall sample inhomogeneity. 


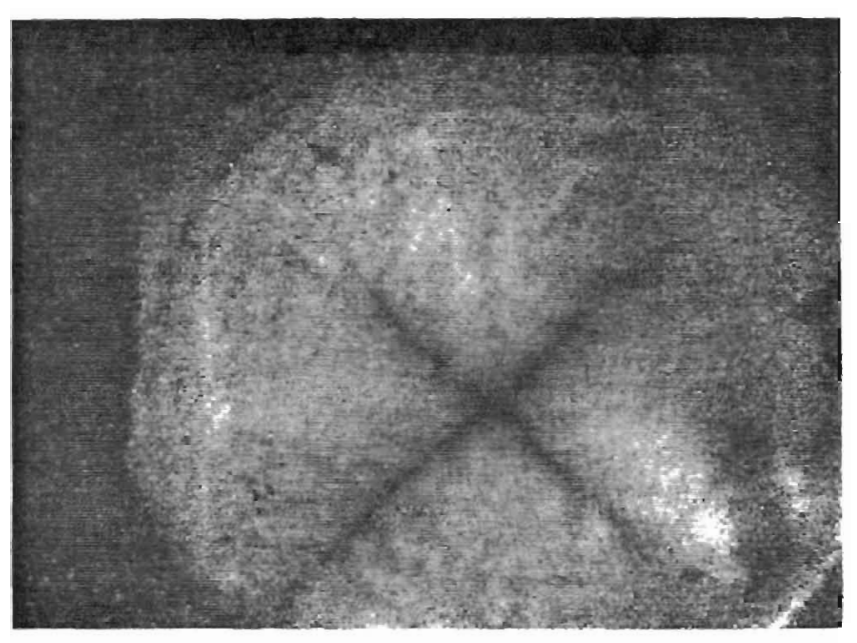

Fig. 5. Flux distribution in an epitaxial $100 \mathrm{~nm}$ thick $\mathrm{YBa}_{2} \mathrm{Cu}_{3} \mathrm{O}_{7-x}$ film on a $10 \times 10 \mathrm{~mm}^{2}$ (100) MgO substrate, after zero field cooling. The externally applied field is $60 \mathrm{mT}$. The low contrast of this picture is due to the rather homogeneous flux distribution

\section{Conclusions}

In summary we have investigated the flux distributions of epitaxial $\mathrm{YBa}_{2} \mathrm{Cu}_{3} \mathrm{O}_{7-x}$ films with a contactless magnetooptic experiment in fields up to $1 \mathrm{~T}$ and in addition with a scanning micro Hall probe in the low field range. A strong influence of the sample geometry on the flux penetration process is observed. In contrast to recent experiments by Forkl et al. [2], we have found the flux to penetrate our samples in a rather regular way, yielding nearly symmetric shielded regions, roughly along the sample di- agonals. In the remanent state the area close 10 the structure appears to be the region where the flux is pinned most effectively. We attribute the observed way of penetration to the influence of the curvature of the current path on the shielding properties. This method appears to be a sensitive check of the homogeneity of superconducting films.

\section{Acknowledgements}

We thank B. Stritzker and P. Berberich for the thin film samples investigated here. This work was supported by the Federal Ministry of Research and Technology (BMFT) of the Federal Republic of Germany, grant number 13N5705, and by the Schwerpunktprogramm Land Baden Württemberg.

\section{References}

[1] M.V. Indenbom, N.N. Kolesnikov, M.P. Kulakov, I.G. Naumenko, V.I. Nikitenko, A.A. Polyanskii, N.F. Vershinin and V.K. Vlasko-Vlasov, Physica C 166 (1990) 486.

[2] A. Forkl, T. Dragon, H. Kronmüller, H.-U. Habermeier and G. Mertens, Appl. Phys. Lett. 57 (1990) 1067.

(3) J. Fröhlingsdorf, W. Zander and B. Stritzker, Solid State Commun. 67 (1988) 965.

[4] H. Kirchner, Phys. Lett. A 30 (1969) 437.

[5] J. Schoenes, Z. Phys. B 20 (1975) 345.

[6] R.P. Huebener, V.A. Rowe and R.T. Kampwirth, J. Appl. Phys. 41 (1970) 2963.

[7] P. Laeng and L. Rinderer, Cryogenics 8 (1972) 315.

[8] H. Kirchner, Phys. Status Solidi (A) 4 (1971) 531.

[9] L.T. Baczewski, K. Piotrowski R. Szymcak and A.P. Malozemoff, Physica C 175 (1991) 363. 